\title{
Assembly of Carbon Nanotubes between Electrodes by Utilizing Optically Induced Dielectrophoresis and Dielectrophoresis
}

\author{
Pei-Fang $\mathrm{Wu}^{1}$ and Gwo-Bin Lee ${ }^{2}$ \\ ${ }^{1}$ Department of Engineering Science, National Cheng Kung University, Tainan 701, Taiwan \\ ${ }^{2}$ Department of Power Mechanical Engineering, National Tsing Hua University, Hsinchu 30013, Taiwan
}

Correspondence should be addressed to Gwo-Bin Lee, gwobin@pme.nthu.edu.tw

Received 1 May 2011; Revised 30 June 2011; Accepted 6 July 2011

Academic Editor: Aaron T. Ohta

Copyright (C) 2011 P.-F. Wu and G.-B. Lee. This is an open access article distributed under the Creative Commons Attribution License, which permits unrestricted use, distribution, and reproduction in any medium, provided the original work is properly cited.

\begin{abstract}
This study reports a new approach for assembling carbon nanotubes (CNTs) between electrodes combination of optically induced dielectrophoresis force and dielectrophoresis force. Metal electrodes and amorphous silicon layer were first patterned and then used to assemble CNTs. By utilizing moving light patterns, the CNTs could be collected to the central area between two metal electrodes. The CNTs with different concentrations can be collected and aligned to form CNTs lines with different widths. The immobilization of preassembled CNTs was also demonstrated by exposing them to an ultraviolet light source such that they can be fixed at the prealigned location. Then photoresist asher was used to get rid of the cured polymer. The development of the new platform can be promising to massively assemble CNTs.
\end{abstract}

\section{Introduction}

The application of CNTs in various fields has increased dramatically and has made a substantial impact recently since their discovery [1]. For example, they are regarded as promising building blocks for next-generation nanosensing devices due to their high-aspect-ratio structures, unique electronic characteristics, and excellent transducing properties $[2,3]$. With advances in micro- and nanotechnologies, these nanosensing devices are capable of metering physical, chemical or biological properties at a micro- or even nanoscale. For instance, CNTs can be trapped and patterned across prefabricated electrode arrays to yield reproducible sensing features in constructing gas sensors $[4,5]$. However, there are several issues involved when patterned CNTs across electrodes to form nanosensors. First, reproducible and wellcontrolled patterns of CNTs must be made such that these nanosensors will behave in a reproducible manner from device to device. Furthermore, parallel assembly of these CNTs is crucial for mass production of any CNT-based nanosensors.

A variety of methods for the manipulation of CNTs have been demonstrated in the literature, including guided
CNTs growth [6, 7], magnetic forces [8, 9], polar molecular patterning [10], and optical tweezers [11]. For instance, organized CNTs structures can be formed by using chemical vapor deposition such that guided CNTs can grow in situ. Alternatively, pregrown CNTs can be aligned and manipulated after they have been formed. However, it is either a lengthy process or remains challenging when attempts are made to align them in parallel between two electrodes for a large-scale fabrication process. Furthermore, a variety of techniques for modifying the surfaces of CNTs may also give rise to self-assembled ordered structure of CNTs on metal substrates [12]. Alternatively, optical tweezers (OTs) have been explored as a flexible method for the manipulation of CNTs [13]. Although OTs can manipulate objects accurately by using high-power laser beams to trap the nanoobjects, they also need a high-precision positioning process for the manipulation of CNTs, hence, hindering their practical applications. Furthermore, the experimental setup for an OT system is relatively costly.

Recently, another technique, dielectrophoresis (DEP) [14], has been commonly used for CNT manipulation. For example, CNTs could be aligned between two metal electrodes with ease by using this approach $[15,16]$. However, 
the number and the total width of clustered CNTs are difficult to be precisely controlled since the induced DEP force exists only in the neighborhood of the electrodes. Therefore, it may significantly affect the density of the CNTbased nanosensors. Consequently, a primary challenge involved in the fabrication of these nanosensing devices is the controllable manipulation of CNTs, and, thus, it still remains challenging to align a well-controlled number of CNTs between two electrodes. Recently, optically induced dielectrophoresis (ODEP) and optoelectronic tweezers (OET) have been reported to manipulate microparticles [17], or even metal nanowires and CNTs $[18,19]$. Furthermore, the manipulation and patterning of CNTs has been demonstrated [20]. The ODEP was further demonstrated as a powerful tool to manipulate CNTs. However, the attempt to assemble CNTs between electrodes to form a CNT-based nanosensor by utilizing ODEP and dielectrophoresis has never been made. In this study, a new method to assemble CNTs on a nanosensor by using the combination of ODEP and DEP forces was reported for the first time. It may allow for large-scale, parallel assembly of CNTs with a well-controlled width across two electrodes, which is crucial for the practical application of CNT-based nanosensors.

\section{Materials and Methods}

2.1. Operating Principle. Figure 1 shows a schematic illustration of the new approach for assembling CNTs across electrodes. Briefly, the DEP force was used to attract more CNTs around the electrodes. Then, CNTs were collected and transported to the central area between the two electrodes by using the ODEP force from a projected moving light pattern. The width of the CNTs line can be controlled by either the collection time or the initial concentration of the suspended CNTs. The chip was composed of an upper indium-tin-oxide (ITO) glass and a bottom substrate coated with a photoconductive layer (hydrogenated amorphous silicon, a-Si) and patterned metal electrodes. The gap spacing between the top and the bottom layer is typically defined using doublesided tape with $30-\mu \mathrm{m}$ thickness. The entire region can induce the ODEP force except the metal electrodes since they are not supplied with alternating current (AC) voltages. When an AC voltage was applied across the metal electrodes patterned on the bottom substrate, a lateral nonuniform electric field can be generated. Afterwards, when a projected light source illuminates the a-Si layer, electron-hole pairs were excited, thus, decreasing the impedance of the a-Si layer by 4 to 5 orders of magnitude. Then the AC voltage dropped across the liquid layer inside the illuminated area such that a non-uniform electric field can be induced. The light-illuminated region can be regarded as the "virtual electrode." When CNTs were suspended in the non-uniform electric field, a positive ODEP force can be induced when an appropriate frequency was applied and thus CNTs can be manipulated and prealigned across the electrodes by moving the light patterns which has been programmed by a computer. Therefore, a thin line of CNTs can be aligned across the electrodes.

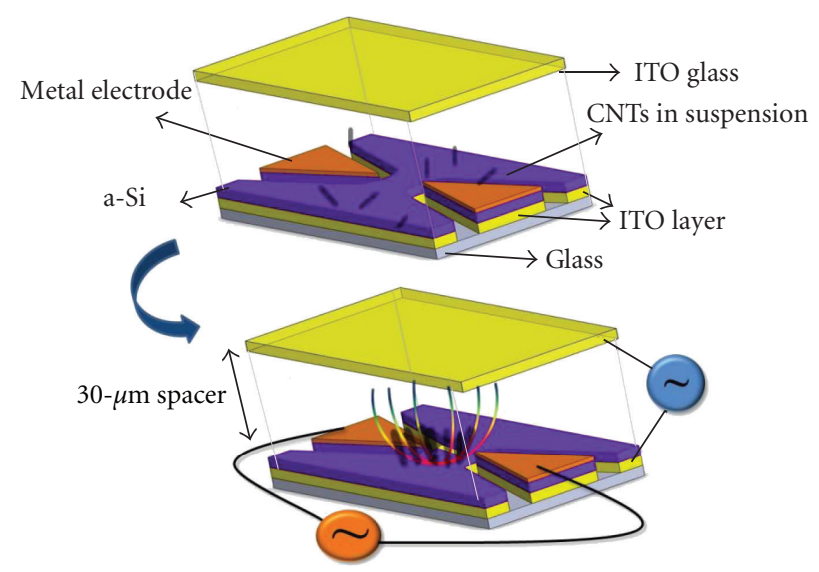

Figure 1: Schematic illustration of the new ODEP platform for assembling CNTs between electrodes to form a CNT-based nanosensor. The ODEP chip was composed of an upper ITO glass and a bottom substrate coated with a photoconductive layer (a-Si) and patterned gold electrodes.

The DEP force for nanotubes with a radius of $r$ and a length of $l$, including CNTs, can be represented as follows using an elongated ellipsoid model [21]:

$$
F=\left(\frac{\pi r^{2} l}{6}\right) \mathcal{E} m \operatorname{Re}[K *(\omega)] \nabla\left(E^{2}\right),
$$

where $l$ denotes the length of the nanotubes, $\varepsilon m$ represents the permittivity of the surrounding medium, $r$ is the radius of the nanotubes, $E$ is the electric field, and $\operatorname{Re}[K *(\omega)]$ is known as the real part of the Clausius-Mossotti (CM) factor. The induced ODEP forces are proportional to the gradient of the square of the electric field strength $\left[\nabla\left(E^{2}\right)\right]$ which is directly related to the efficiency of the photoconductivity effect of the a-Si layer.

2.2. Fabrication Process. Figure 3 shows a schematic illustration of the simplified fabrication process for the ODEP chip. The chip is with typical sizes of $4 \mathrm{~cm} \times 4 \mathrm{~cm}$. A 10 $\mathrm{nm}$ thick molybdenum layer, acting as an adhesive and conductive layer, was first deposited onto a 1-mm thick ITO glass substrate. A $0.5-\mu \mathrm{m}$ thick a-Si was then deposited on top of the adhesive layer by using a plasma-enhanced chemical vapor deposition (PECVD) process. Then, the a-Si layer was patterned by using a reactive ion etching process. Next, a standard wet ITO etching process was used to pattern ITO electrodes. The purpose of this step was to isolate the ITO electrodes from the photoconductive material layer. Next, the fabrication process involved the deposition of an adhesion layer of $150-\AA \AA$ chrome and a $1000-\AA$ gold electrode layer by using an electron-beam evaporation process. Photolithography was then used to pattern the metal layer into a triangular shape. The two electrodes face inward with a tip-to-tip distance of $70 \mu \mathrm{m}$. ODEP and DEP forces can be induced when two AC voltages were applied at different electrodes.

2.3. Experimental Setup. CNTs were first dispersed in a $10-\mathrm{mL}$ solution containing $20 \% \mathrm{w} / \mathrm{v}$ poly(ethylene glycol) 


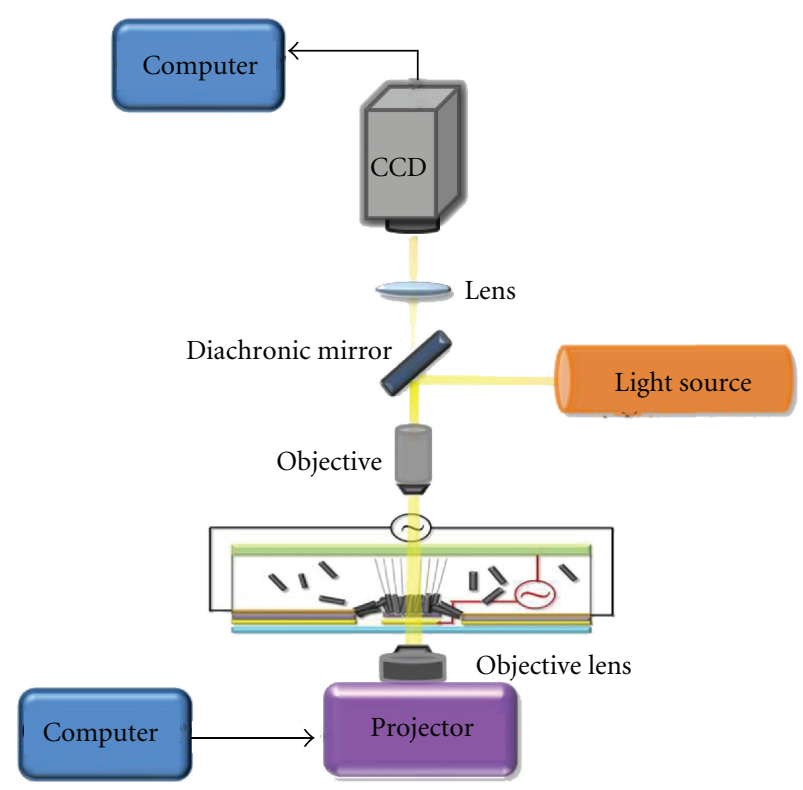

(a)
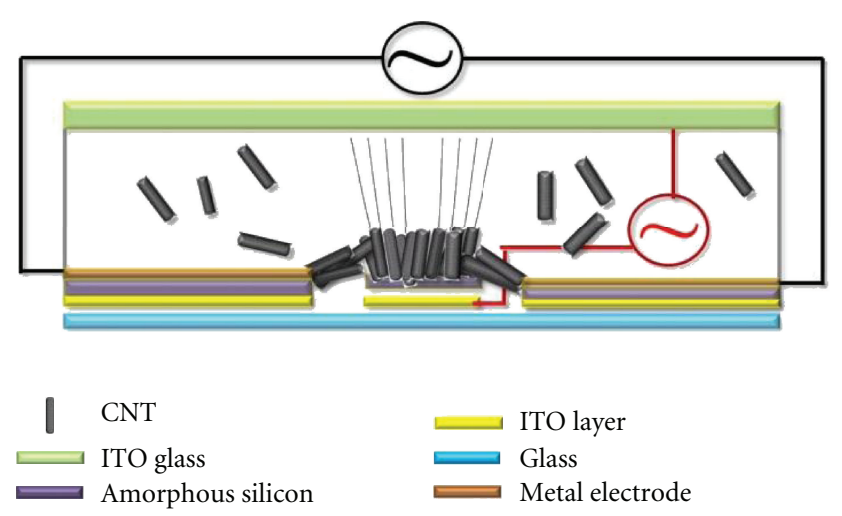

(b)

FIGURE 2: Schematic illustration of the experimental setup and the assembly process of CNTs between ITO electrodes by using a positive ODEP force and a DEP force.

diacrylate (PEGDA) and $0.2 \% \mathrm{w} / \mathrm{v}$ photoinitiator (Irgacure 2959) [18] so that the electrical conductivity of the solution formed was around $80 \mu \mathrm{S} / \mathrm{cm}$. PEGDA is an ultraviolet (UV)curable polymer $[22,23]$ that forms a three-dimensional polymer matrix in the presence of an UV source. CNTs with purity not less than $90 \%$ were chosen as the solute in this study. Since CNTs are hydrophobic and tend to bundle together, it was difficult to achieve well-dispersed CNTs. After mixing, the CNTs solution was bath sonicated for 1 hour to become more dispersed CNTs and then centrifuged for $3 \mathrm{~min}$ at $1500 \mathrm{rpm}$. Around $5 \mu \mathrm{L}$ of the solution was then pipetted into the ODEP chip.

Figure 2 shows the experimental setup. A digital projector (PJ1172, Viewsonic, Japan) was used to illuminate preprogrammed light patterns as virtual electrodes onto the ODEP platform. A 50x objective lens (Nikon, Japan) was fixed between the projector and the ODEP chip to focus the projected light onto the chip to form light patterns. It was

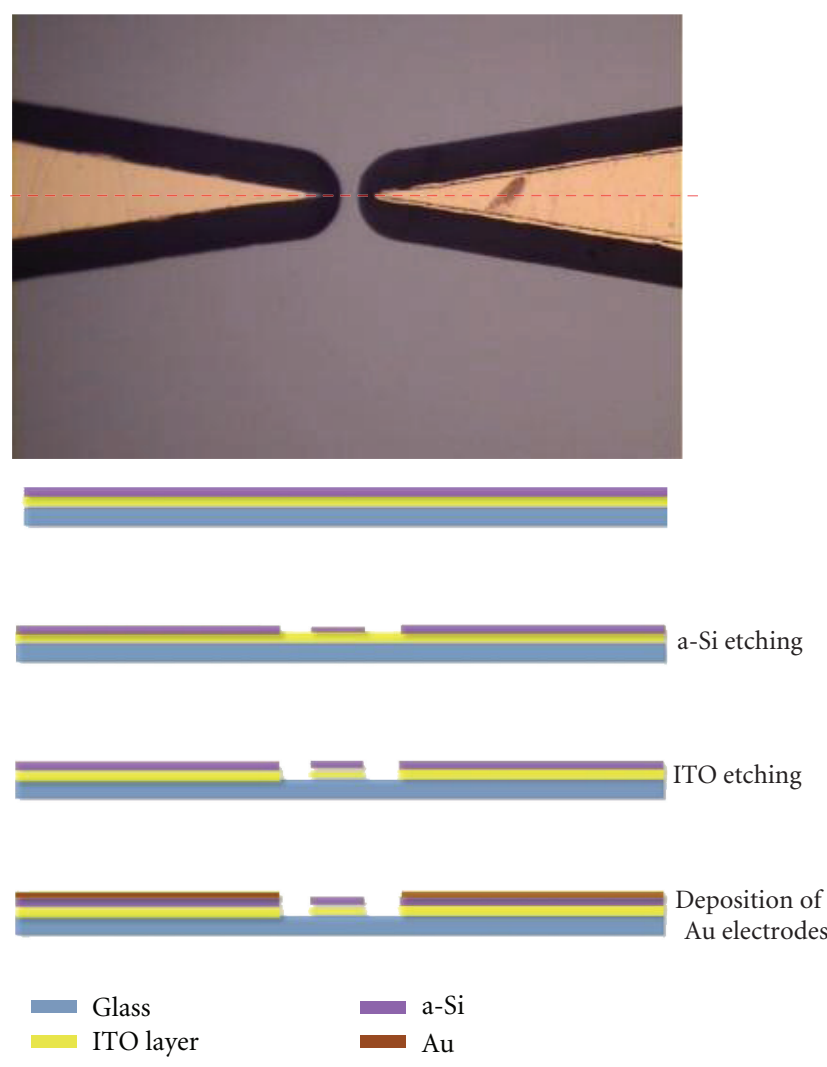

FIGURE 3: The simplified fabrication process of the ODEP chip.

composed of a charge-coupled device (CCD) camera (SSCDC80, Sony, Japan), an optical microscope (Zoom 125C, OPTEM, USA), and a computer equipped with an image acquisition interface card. The AC voltage was supplied by using two function generators (Model 195, Wavetek, UK; AFG310 TEKTRONIX, USA), and a power amplifier (790 Series, AVC Instrumentation, USA). In this study, an AC power source with a frequency of $100 \mathrm{kHz}$ and a magnitude of $20 \mathrm{Vpp}$ was applied across the top and bottom layers of the ODEP chip to generate an electrical field. Similarly, the voltage applied across the metal electrodes was $30 \mathrm{Vpp}$ with a frequency of $110 \mathrm{kHz}$. Note that the operating frequencies of these two applied voltages must be different. If the two applied frequencies were the same, the CNTs connected to the electrodes would move up and down, which is a result of the interference between the simultaneous DEP and ODEP forces.

\section{Results and Discussion}

As mentioned previously, moving light patterns can be used to attract and collect dispersed CNTs in the medium to the center of the electrodes when using ODEP forces. Figure 4 shows a series of photographs for prealigned CNTs at the electrodes. First, an AC potential was applied to the metal electrodes to generate the required electric field for the DEP alignment process (Figure 4(a)). The CNTs would adhere to the electrodes temporarily. Second, the CNTs 


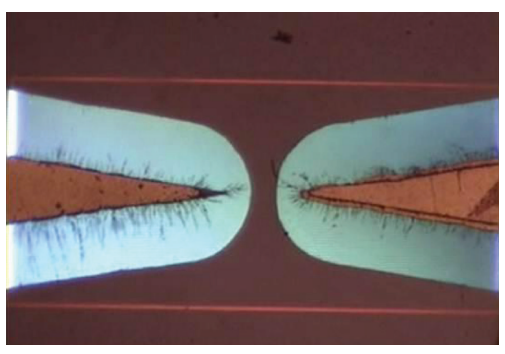

(a)

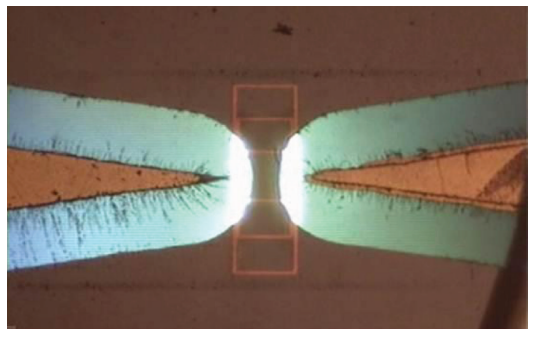

(d)

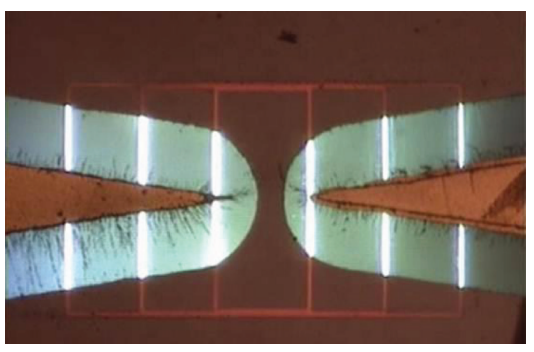

(b)

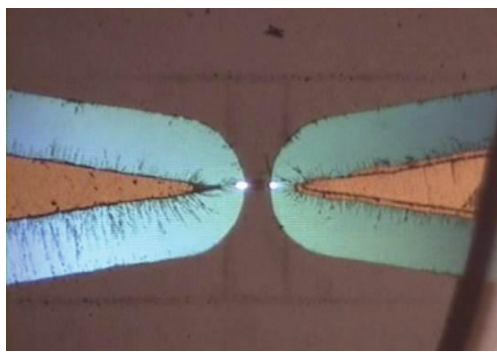

(e)

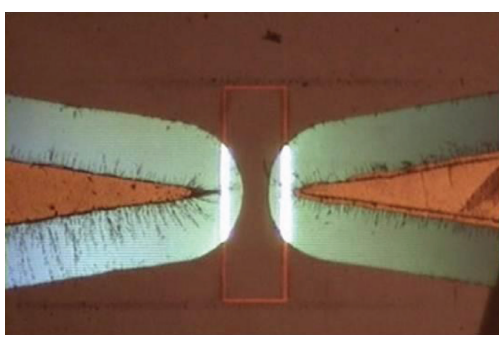

(c)

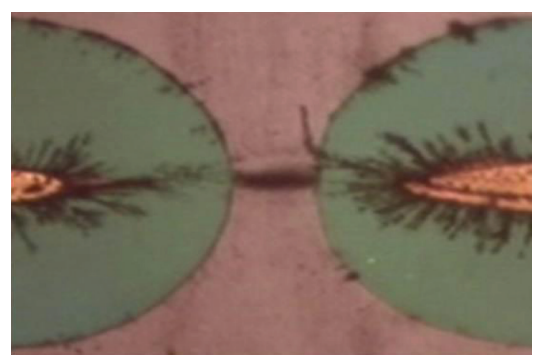

(f)

FIGURE 4: A series of snapshot photographs for collecting CNTs to the central area between metal electrodes by using moving optical images and DEP forces.

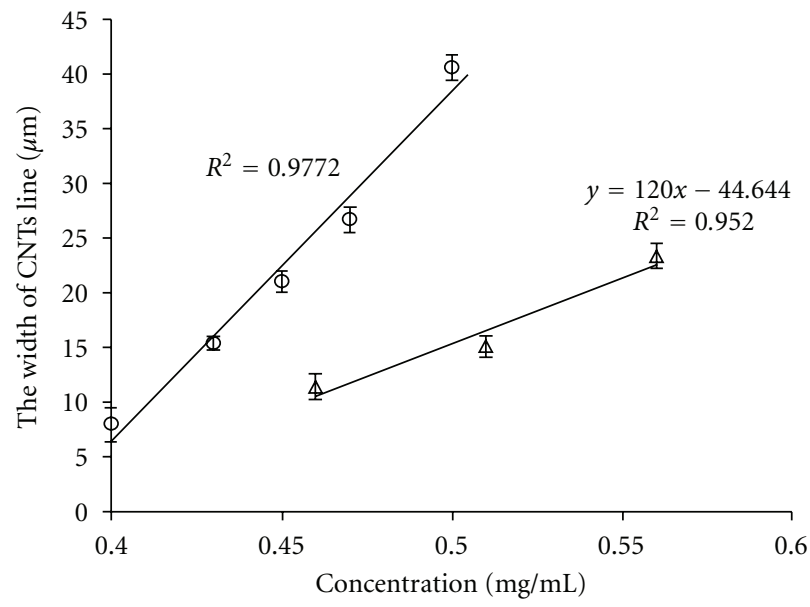

○ ODEP

$\triangle$ ODEP and DEP

FIGURE 5: The relationship between the width of the line of CNTs and the concentration of the CNTs.

were successfully collected by using the ODEP force. CNTs were attracted and collected to a specific area through the manipulation of the moving optical images designed by the computer program (Figures 4(b)-4(e)). A specific light track to collect suspended CNTs was generated as follows. First, six vertical lines inside a big square were used to collect CNTs. Then, these six lines moved toward the center and then collapsed into two lines to define the width of the CNTs line between two electrodes such that the suspended CNTs were collected horizontally. Finally, another six horizontal lines were generated to collect the suspended CNTs. These six lines moved and then collapsed again to only one line. With this approach, suspended CNTs can be collected and aligned into a thin line across two electrodes. The patterns of the light track were flexible and could be finetuned based on the required line width. Note that the moving speed of the light track should be controlled appropriately. If the light track moved too fast, the CNTs cannot move with the light track so that the collected CNTs may not be enough.

It was clearly observed that most of the CNTs in the manipulation area can be successfully collected by animated optical images. In this case, the concentration of the CNTs was $0.51 \mathrm{mg} / \mathrm{mL}$, and the resulting line width of the CNTs was measured to be $15 \mu \mathrm{m}$ by utilizing both the DEP and ODEP forces (Figure 4(f)). The operating times for applying the DEP and ODEP forces were five minutes and three minutes, respectively. It was found that the line width of the CNTs can be adjusted by controlling the initial concentrations of CNTs. Figure 5 shows the relationship between the width of CNTs lines and the concentrations of the CNTs at an applied voltage of $20 \mathrm{Vpp}$. It can be clearly observed that at higher concentrations, the width of the line of CNTs line increases. Note that lower initial CNT concentrations were tested. However, the line of CNTs cannot be formed at concentrations lower than $0.45 \mathrm{mg} / \mathrm{mL}$, indicating that the narrowest width of the nanosensor was $8 \mu \mathrm{m}$ in our current experimental configuration. The width of CNT line also depended on the light width from the pro-jector. The minimum line width provided by this ODEP plat-form was approximately $5 \mu \mathrm{m}$. By adopting a higher power projector and a better optical setup, this minimum line width could be further minimized.

Nevertheless, utilizing the ODEP force only cannot connect the CNTs to the electrodes. Hence, the DEP force was 


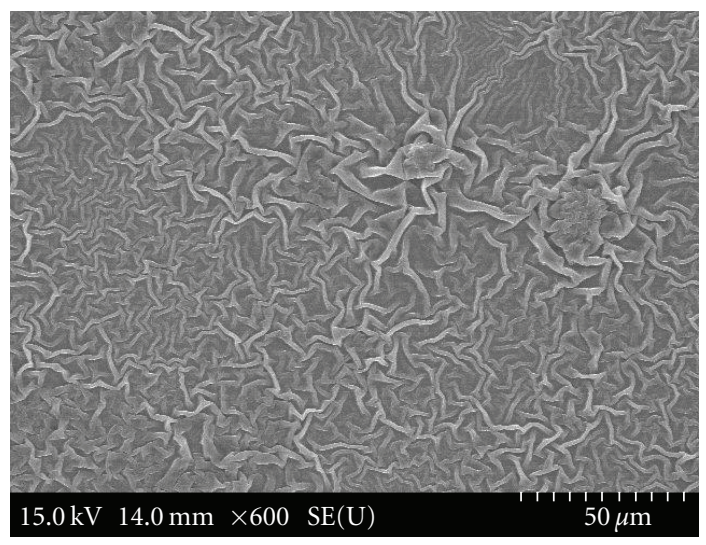

(a)

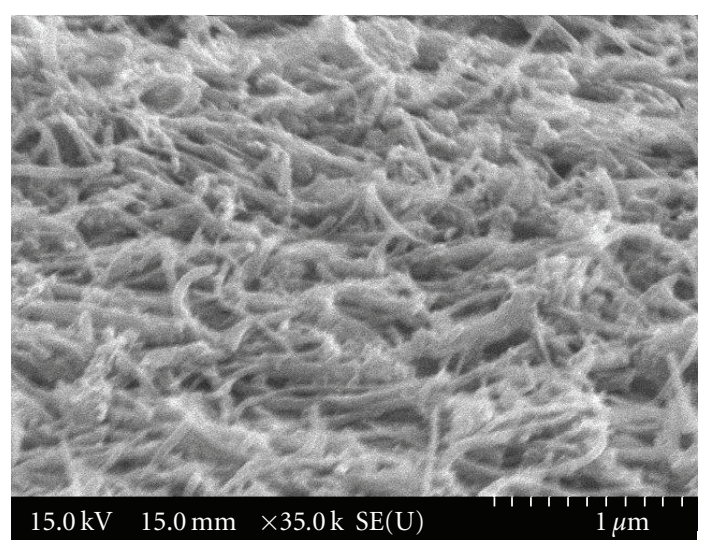

(b)

Figure 6: SEM images of CNTs in the photoresist (a) before and (b) after the ashing process.

further used before the CNTs were collected. The relationship between the width of the CNT lines and the concentrations of the CNTs using a combination of the ODEP and DEP is also shown in Figure 5. Some CNTs would connect to the electrode when using DEP so that the CNTs in the neighboring area would decrease. Consequently, the line width will be narrower if utilizing the DEP and the ODEP simultaneously at the same concentration. The fewer number of CNTs connected, the narrower the resulting line width. The coefficients of variation for the measurements of the widths were $10 \%, 4 \%$, and $6 \%$ for CNTs concentrations of $0.45,0.51$, and $0.56 \mathrm{mg} / \mathrm{mL}$, respectively, indicating that the CNT-based nanosensors can be fabricated by using the proposed method with good reproducibility.

Another issue after the alignment of the CNTs is to fix them to the original location. As mentioned previously, an UV illumination (UV-A) through a metal mask for 10 minutes was used to immobilize the CNTs once the nanowires were positioned at the desired location. The UVcurable polymer was then removed by a photoresist asher such that the prealigned CNTs can be used. Note that the time and the power of the photoresist asher need to be controlled carefully to avoid the oxidation and damage of the prealigned CNTs. Figure 6(a) shows a scanning electron microscopy (SEM) image of CNTs in the photoresist before using the ashing process. After the ashing process, most of the polymers were ashed and the CNTs were exposed for the subsequent sensing applications (Figure 6(b)).

\section{Conclusion}

This study, for the first time, reports a new approach for assembly of carbon nanotubes between electrodes by utilizing optically induced dielectrophoresis and the dielectrophoresis forces. An animated optical pattern was designed to collect the CNTs in the medium. The widths of the CNTs were reasonably controlled. The minimum line width of the CNTs was measured to be $8 \mu \mathrm{m}$. Furthermore, the prealigned CNTs were immobilized by exposure to an UV source. This developed platform may be promising for controlling the width of CNTs in nanosensors.

$\begin{array}{ll}\text { Abbreviation and Nomenclature } \\ \text { AC: } & \text { Alternating current } \\ \text { a-Si: } & \text { Hydrogenated amorphous silicon } \\ \text { CCD: } & \text { Charge-coupled device } \\ \text { CM: } & \text { Clausius-Mossotti factor } \\ \text { CNTs: } & \text { Carbon nanotubes } \\ \text { DEP: } & \text { Dielectrophoretic } \\ E: & \text { Intensity of the applied electric field } \\ \varepsilon m: & \text { Electrical permittivity of the } \\ & \text { surrounding medium } \\ \text { Fdep: } & \text { Dielectrophoretic force } \\ \text { ITO: } & \text { Indium-tin-oxide } \\ l: & \text { Length of the nanotubes } \\ \text { ODEP: } & \text { Optically induced dielectrophoresis } \\ \text { OET: } & \text { Optoelectronic tweezers } \\ \text { OT: } & \text { Optical tweezers } \\ \text { PEGDA: } & \text { Poly(ethylene glycol) diacrylate } \\ r: & \text { Radius of the nanotubes } \\ \text { Re[K } *(\omega)]: \text { Real part of Clausius-Mossotti factor } \\ \text { SEM: } & \text { Scanning electron microscopy. } \\ & \end{array}$

\section{Acknowledgment}

The authors gratefully acknowledge the financial support provided to this study by the National Science Council in Taiwan (NSC 96-2120-M-007-015).

\section{References}

[1] S. Iijima, "Helical microtubules of graphitic carbon," Nature, vol. 354 , no. 6348 , pp. 56-58, 1991.

[2] D. Tasis, N. Tagmatarchis, A. Bianco, and M. Prato, "Chemistry of carbon nanotubes," Chemical Reviews, vol. 106, no. 3, pp. 1105-1136, 2006.

[3] A. Jorio, M. S. Dresselhaus, and G. Dresselhaus, Carbon Nanotubes, Springer, Berlin, Germany, 2008.

[4] J. Suehiro, G. Zhou, and M. Hara, "Fabrication of a carbon nanotube-based gas sensor using dielectrophoresis and its application for ammonia detection by impedance spectroscopy," Journal of Physics D, vol. 36, no. 21, pp. L109-L114, 2003. 
[5] M. Trojanowicz, "Analytical applications of carbon nanotubes: a review," Trends in Analytical Chemistry, vol. 25, no. 5, pp. 480-489, 2006.

[6] B. Q. Wei, R. Vajtai, Y. Jung et al., "Organized assembly of carbon nanotubes," Nature, vol. 416, no. 6880, pp. 495-496, 2002.

[7] J. Hu, M. Ouyang, P. Yang, and C. M. Lieber, "Fabricating carbon nanotube transistor device," Nature, vol. 399, pp. 48-51, 1999.

[8] J. E. Fischer, W. Zhou, J. Vavro et al., "Magnetically aligned single wall carbon nanotube films: preferred orientation and anisotropic transport properties," Journal of Applied Physics, vol. 93, no. 4, pp. 2157-2163, 2003.

[9] Y. Huang, X. Duan, Q. Q. Wei, and C. M. Lieber, “Directed assembly of one-dimensional nanostructures into functional networks," Science, vol. 291, no. 5504, pp. 630-633, 2001.

[10] S. G. Rao, L. Huang, W. Setyawan, and S. Hong, "Large-scale assembly of carbon nanotubes," Nature, vol. 425, no. 6953, pp. 36-37, 2003.

[11] D. G. Grier, "A revolution in optical manipulation," Nature, vol. 424, no. 6950, pp. 810-816, 2003.

[12] Z. Liu, Z. Shen, T. Zhu et al., "Organizing single-walled carbon nanotubes on gold using a wet chemical self-assembling technique," Langmuir, vol. 16, no. 8, pp. 3569-3573, 2000.

[13] S. Tan, H. A. Lopez, C. W. Cai, and Y. Zhang, "Optical trapping of single-walled carbon nanotubes," Nano Letters, vol. 4, no. 8, pp. 1415-1419, 2004.

[14] H. W. Seo, C. S. Han, D. G. Choi, K. S. Kim, and Y. H. Lee, "Controlled assembly of single SWNTs bundle using dielectrophoresis," Microelectronic Engineering, vol. 81, no. 1, pp. 83-89, 2005.

[15] S. Tung, H. Rokadia, and W. J. Li, "A micro shear stress sensor based on laterally aligned carbon nanotubes," Sensors and Actuators A, vol. 133, no. 2, pp. 431-438, 2007.

[16] A. Vijayaraghavan, S. Blatt, D. Weissenberger et al., "Ultralarge-scale directed assembly of single-walled carbon nanotube devices," Nano Letters, vol. 7, no. 6, pp. 1556-1560, 2007.

[17] P. Y. Chiou, A. T. Ohta, and M. C. Wu, "Massively parallel manipulation of single cells and microparticles using optical images," Nature, vol. 436, no. 7049, pp. 370-372, 2005.

[18] A. Jamshidi, P. J. Pauzauskie, P. J. Schuck et al., "Dynamic manipulation and separation of individual semiconducting and metallic nanowires," Nature Photonics, vol. 2, no. 2, pp. 86-89, 2008.

[19] P. J. Pauzauskie, A. Jamshidi, J. K. Valley, J. H. Satcher, and M. C. Wu, "Parallel trapping of multiwalled carbon nanotubes with optoelectronic tweezers," Applied Physics Letters, vol. 95, no. 11, Article ID 113104, 3 pages, 2009.

[20] M. W. Lee, Y. H. Lin, and G. B. Lee, "Manipulation and patterning of carbon nanotubes utilizing optically induced dielectrophoretic forces," Microfluidics and Nanofluidics, vol. 8, no. 5, pp. 609-617, 2010.

[21] T. B. Jones, Electromechanics of Particles, Cambridge University Press, Cambridge, UK, 1995.

[22] D. J. Beebe, J. S. Moore, J. M. Bauer et al., "Functional hydrogel structures for autonomous flow control inside microfluidic channels," Nature, vol. 404, no. 6778, pp. 588-590, 2000.

[23] M. Law, L. E. Greene, J. C. Johnson, R. Saykally, and P. Yang, "Nanowire dye-sensitized solar cells," Nature Materials, vol. 4, no. 6, pp. 455-459, 2005. 

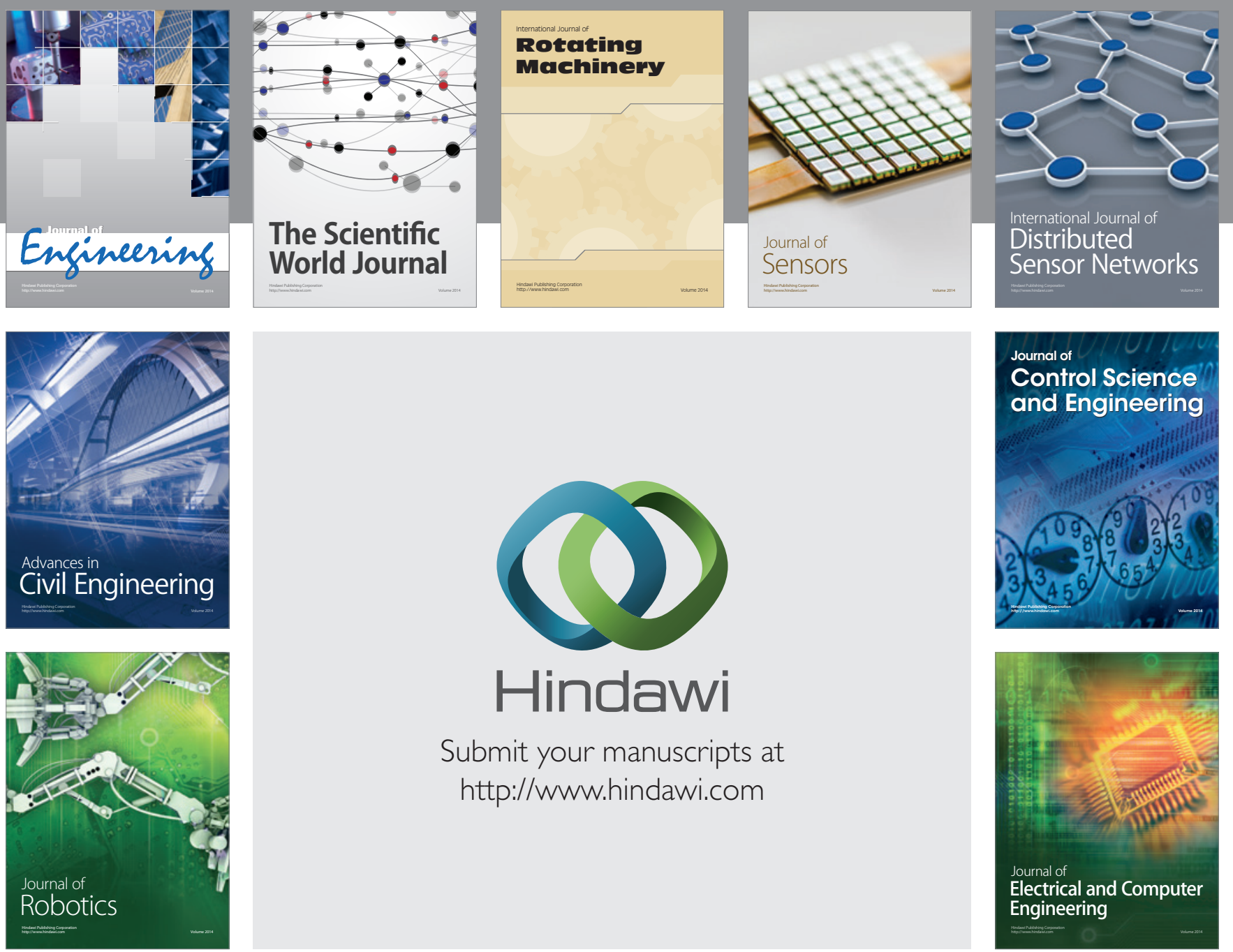

Submit your manuscripts at

http://www.hindawi.com
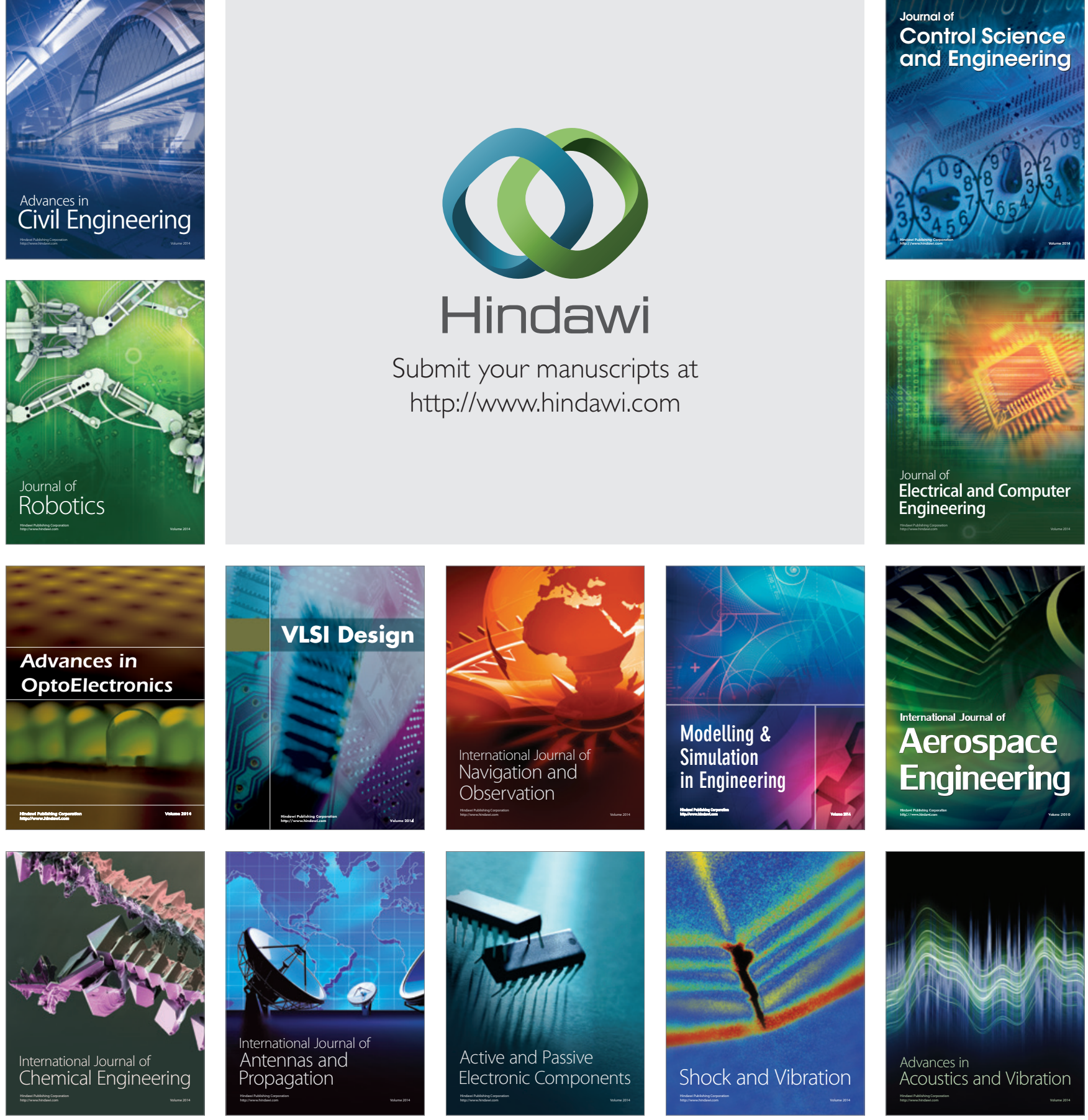